\title{
A Novel Viscous Throughflow Model for Compressor Analysis and Its Application
}

\author{
Hailiang Jin", Daobin Qiu, Yueqian Yin \\ AECC Hunan Aviation Powerplant Research Institute, Hunan Key Laboratory of Turbomachinery on Small and Medium Aero-Engine, \\ Zhuzhou, China \\ Email address: \\ jinhailiang1984@gmail.com (Hailiang Jin),936007974@qq.com (Daobin Qiu), enginee3562@sina.com (Yueqian Yin) \\ ${ }^{*}$ Corresponding author
}

\section{To cite this article:}

Hailiang Jin, Daobin Qiu, Yueqian Yin. A Novel Viscous Throughflow Model for Compressor Analysis and Its Application. International Journal of Fluid Mechanics \& Thermal Sciences. Special Issue: Fluid Mechanics \& Thermal Sciences in Turbomachines.

Vol. 6, No. 3, 2020, pp. 89-94. doi: 10.11648/j.ijfmts.20200603.13

Received: July 12, 2020; Accepted: August 25, 2020; Published: September 7, 2020

\begin{abstract}
Throughflow calculations are still an inevitable step in the aerodynamic design of compressors. The viscous throughflow model derived from Navier-Stokes equations can be more capable in predicting choked flow and capturing shock waves compared to the traditional methods. In this paper, authors further developed the inviscid model for a previously developed throughflow analysis method. To obtain the governing equations, three-dimensional Navier-Stokes equations combined with the Spalart-Allmaras turbulence model were circumferentially averaged with the assumption that the flow was circumferentially uniform. A viscous blade force and an inviscid blade force had been calculated. The Miller's correlations of deviation angle and loss were incorporated to model these forces. The governing equations are discretized by an explicit fourstep Runge-Kutta scheme and solved by a time-marching finite volume method. Current model was verified through predicting the performances of a 1.5 stage fan. The agreements between the experiments and calculations are reasonably good. This throughflow model can predict quite similar flow patterns and radial profiles of some parameters compared to a CFD software, which shows the potential of this model. There are still some notable deviations between the results from throughflow analysis and that from CFD calculation. Future work is to improve the prediction of deviation angle and loss near the endwall regions.
\end{abstract}

Keywords: Throughflow Model, Viscous, Compressor, Analysis, Application

\section{Introduction}

Recently, the precision of Computational Fluid Dynamics (CFD) method has been largely improved, and the rapid growth of computing capability makes it much less timeconsuming to perform complex CFD analysis. Therefore, CFD analysis begins to play a more important role in the aerodynamic design of compressors. However, the throughflow calculation is still the base of the whole design process, as a crucial step during the preliminary design stage. The throughflow method is utilized to determine the velocity triangles and predict the meridional flow fields of the compressor. A well-developed, high-precision throughflow model facilitates the design by remarkably reducing the iterative steps during the design process.

The streamline curvature method, for instance, is one of the most common and effective approaches in the aerodynamic design of turbomachinery [1, 2]. Spurr then proposed a throughflow model by circumferentially averaging Navier-Stokes equations [3]. This model can predict the choked flow, capture shock waves in blade passages, and cope with subsonic or supersonic flows. Therefore, such viscous throughflow methods can be more powerful compared to traditional streamline curvature method, and merits continues development.

In this paper, the author also presents a viscous throughflow model. Firstly, the approach to obtain governing equations is described. The three-dimensional Navier-Stokes equations combined with the Spalart-Allmaras turbulence model were circumferentially averaged with the assumption that the flow was circumferentially uniform. Next, the inviscid blade force and viscous blade force models used in this throughflow model are briefly described. Subsequently, the numerical method to solve the governing equations are 
also presented. Finally, this throughflow model is applied to predict the performance of an advanced transonic fan, and comparisons among several methods are made.

\section{Throughflow Model}

\subsection{Governing Equations}

The governing equations of the throughflow model in the relative frame of reference are formulated as follows:

$$
\frac{\partial U}{\partial t}+\frac{1}{b r} \frac{\partial\left[b r\left(F-F_{v}\right)\right]}{\partial x}+\frac{1}{b r} \frac{\partial\left[b r\left(G-G_{v}\right)\right]}{\partial r}=S+F_{B}+F_{F}
$$

where the conservative variable vector $U$, convective flux vector $F, F_{v}, G$ and $G_{v}$, source vector $S$, inviscid blade force $F_{B}$ and viscous blade force $F_{F}$ are defined as follows.

$$
\begin{aligned}
& U=\left[\begin{array}{c}
\rho \\
\rho w_{x} \\
\rho w_{r} \\
\rho w_{\varphi} \\
\rho E \\
\rho \tilde{v}
\end{array}\right], F=\left[\begin{array}{c}
\rho w_{x} \\
\rho w_{x} w_{x}+p \\
\rho w_{r} w_{x} \\
\rho w_{x} w_{\varphi} \\
\rho H w_{x} \\
\rho \tilde{v} w_{x}
\end{array}\right], G=\left[\begin{array}{c}
\rho w_{r} \\
\rho w_{x} w_{r} \\
\rho w_{r} w_{r}+p \\
\rho w_{\varphi} w_{r} \\
\rho H w_{r} \\
\rho \tilde{v} w_{r}
\end{array}\right], \\
& F_{v}=\left[\begin{array}{c}
0 \\
\tau_{x x} \\
\tau_{x r} \\
\tau_{\varphi x} \\
\tau_{x x} w_{x}+\tau_{x x} w_{r}+\tau_{\varphi x} w_{u}-q_{x} \\
0
\end{array}\right], G_{v}=\left[\begin{array}{c}
0 \\
\tau_{x r} \\
\tau_{r r} \\
\tau_{r \varphi} \\
\tau_{x r} w_{x}+\tau_{r r} w_{r}+\tau_{r \varphi} w_{\varphi}-q_{r} \\
0
\end{array}\right] \text {, } \\
& S=\left[\begin{array}{c}
0 \\
0 \\
\frac{\rho\left(w_{\varphi}+\omega r\right)^{2}+p-\tau_{\varphi \varphi}}{r} \\
\frac{-\rho w_{r}\left(w_{\varphi}+2 \omega r\right)+\tau_{r \varphi}}{r} \\
\rho w_{r} \omega^{2} r \\
\mathrm{~S}_{t b}
\end{array}\right], F_{B}=\left[\begin{array}{c}
0 \\
\rho f_{B x} \\
\rho f_{B r} \\
\rho f_{B \varphi} \\
0 \\
0
\end{array}\right], F_{F}=\left[\begin{array}{c}
0 \\
\rho f_{F x} \\
\rho f_{F r} \\
\rho f_{F \varphi} \\
0 \\
0
\end{array}\right]
\end{aligned}
$$

The term $b$ is a blockage factor due to the circumferential blade thickness and takes the form

$$
b=1-\frac{\varphi_{s}-\varphi_{p}}{2 \pi / N}
$$

where $\left(\varphi_{p}-\varphi_{s}\right)$ represents the circumferential blade thickness, and $N$ is the number of blades. $\mathrm{S}_{t b}$ denotes the source term of the Spalart-Allmaras turbulence model and the details can be seen in [4].

$$
\begin{aligned}
& \mathrm{S}_{t b}=C_{b 1}\left(1-f_{t 2}\right) \rho \tilde{S} \tilde{v}+1.5\left\{\nabla \cdot\left[(1+\rho \tilde{v}) \mu_{l}\right] \nabla \tilde{v}+C_{b 2} \rho \mu_{l} \nabla \tilde{v} \cdot \Delta \tilde{v}\right\} \\
& -\frac{\rho}{\mu_{l}}\left(C_{w 1} f_{w}-\frac{C_{b 1}}{\kappa^{2}}\right)\left(\frac{\tilde{v} \mu_{l}}{d}\right)^{2}+f_{t 1} \rho \Delta U^{2}
\end{aligned}
$$

circumferentially averaging the Navier-Stokes equations combined with the Spalart-Allmaras turbulence model equation, and neglecting the circumferentially non-uniform terms $[5,6]$.

\subsection{Inviscid Blade Force and Viscous Blade Force Models}

The inviscid and viscous blade forces are responsible for the flow deflection and losses respectively, and thus need to be modeled.

One of the key elements of this throughflow model is the inviscid blade force term. The most widely used approach to model the force is to treat it as an additional time-dependent unknown. Several equations suitable for direct or inverse problems been proposed to model the inviscid blade force [79]. However, solving these equations often suffers from convergence issues, particularly, when an inappropriate relaxation factor is specified. Therefore, this model incorporates a variant of the large-particle method $[10,11]$ to avoid convergence problems.

This method splits one time step into two sub-steps. In the first sub-step, the momentum equations are solved without the inviscid blade force terms, which means the effects caused by the inviscid blade force in the momentum equations are neglected. In the final sub-step, only the effects caused by the inviscid blade force are taken into account in the momentum equations. It means that the component of the momentum, which is normal to the average stream surface, takes a nonzero value at the first sub-step, then it will acquire a zero value at the second sub-step due to the drive effects of the inviscid blade force. This method can be expressed as the following equation,

$$
(\rho \vec{w})^{(2)}=(\rho \vec{w})^{(1)}-\vec{n}\left[(\rho \vec{w})^{(1)} \cdot \vec{n}\right]
$$

where $(\rho \vec{w})^{(1)}$ is the momentum computed in the first sub-step, $(\rho \vec{w})^{(2)}$ is the final momentum at the end of this time step, and $n$ is the normal vector for the average stream surface.

The viscous blade force is related to the blade wall skin friction effects on the passage flow. And it can be modeled by the distributed loss model [12]. And the final expression of the viscous blade force is formulated by

$$
\overrightarrow{f_{F}}=-T \frac{w_{m}}{w} \frac{\partial s}{\partial m} \frac{\vec{w}}{w}
$$

where $w_{m}$ is the meridional velocity, $m$ is the meridional coordinate, and $\frac{\partial s}{\partial m}$ is the gradient of the entropy along the meridional coordinate. Deviation angle and loss are needed both in the inviscid blade force and viscous blade force models. The Miller's correlations [13-15] for deviation angle and loss are used in the blade force models. The details of the inviscid blade force and viscous blade force models can be seen in the author's previous work [16].

The above system of equations can be obtained by 


\section{Numerical Methods}

Governing equations are solved by the time-marching finite volume method. The Edwards' LDFSS scheme is used to discretize the convective fluxes. The details of the Edwards' LDFSS scheme can be referred in [17]. An explicit four-step Runge-Kutta scheme is utilized after the spatial discretization [18]. Total pressure, total temperature and velocity direction are specified as inlet boundary conditions. Static pressure is specified as the outlet boundary condition. The no-slip condition is applied at solid boundaries. The detailed solving process to the governing equations are described in the author's previous work [16].

\section{Results}

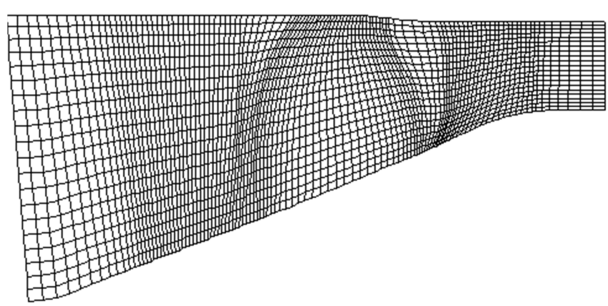

Figure 1. Computational gird of ATS-2 for Euler throughflow calculation.

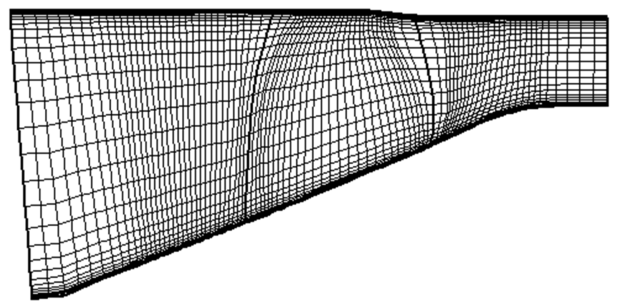

Figure 2. Computational gird of ATS-2 for $N-S$ throughflow calculation.

This throughflow model has been applied to predict the performance of a transonic fan, ATS-2. ATS-2 is an advanced
1.5 stage fan which features a high loading, low aspect ratios and complex three-dimensional swept shapes. To make comparisons, a previously developed inviscid (Euler) throughflow model and a 3D CFD software are also used to predict the performance. The computational meshes for Euler and N-S throughflow calculations are shown in Figure 1 and Figure 2 respectively. The former one is consisted of $111 \times 21$ grid points, $26 \times 21$ of which are in the IGV domain, $30 \times 21$ of which are in the rotor domain, and the rest are in the stator domain. The mesh for N-S calculation has the same grid points in the axial direction, but the grid points near the endwall region have been fined in radial direction. The Carter's rule is applied to determine the deviation angle in the throughflow calculation. The 3D CFD calculation is performed through the Numeca software with the SpalartAllmaras turbulence model, which is also used in the N-S throughflow analysis.

Table 1 shows the overall performance predicted by the Euler throughflow model, N-S throughflow model and Numeca software. The experiment data are also presented in the table. Overall, the throughflow models provide good precision in predicting aerodynamic performance, compared to the experiment data. In terms of the mass flow, all the throughflow models and Numeca software anticipate a lower value compared to the experiment data. Regarding the total pressure ratio, Euler throughflow model gives the highest value among all the methods. However, the adiabatic efficiency obtained from N-S throughflow calculation is the lowest, while the efficiency from the experiment data is the highest. The reason why N-S throughflow model predicts lower mass flow, pressure ratio, adiabatic efficiency than Euler throughflow does is that N-S throughflow models the blockage effects caused by annulus boundary layer.

Table 1. Comparison of global performances of ATS-2 at near design condition.

\begin{tabular}{llll}
\hline \multirow{2}{*}{ Method } & Overall performance & & \\
\cline { 2 - 4 } & Mass flow (kg/s) & Total pressure ratio & Adiabatic efficiency \\
\hline Euler throughflow & 26.112 & 2.2276 & 0.8723 \\
N-S throughflow & 25.914 & 2.2168 & 0.8568 \\
NUMMECA & 25.471 & 2.2074 & 0.8673 \\
Experiment & 26.710 & 2.1990 & 0.8778 \\
\hline
\end{tabular}

Figure 3 and Figure 4 shows the relative Mach number contours of the meridional plane from the Euler and N-S throughflow model at the aerodynamic design point, and Figure 5 is the contours of circumferentially averaged relative Mach number from the 3D CFD analysis. It is notable that the contours of the relative Mach number in Figure 3, Figure 4 and Figure 5 have quite similar patterns. However, there still are some discrepancies among these three figures.

The dense contours in Figure 3 and Figure 4 indicate that a shock is captured near the leading edge of the rotor, resulting high gradients of Mach number, but the gradients are much lower in Figure 5 at the same position. Similar contour lines can be found in the stator domain in Figure 3 and 4, which shows the rapid acceleration downstream the leading edge, this effect is less obvious in Figure 5. In Figure 4, there are regions with low Mach number near the lower and upper endwall, which shows the effects of annulus boundary layer. There is also a low Mach number region near the tip of the rotor in Figure 5, which demonstrates the effect of the tip leakage flow. Although both the Euler and N-S throughflow models do not incorporate such a clearance model, the throughflow model can provide a relatively reasonable meridional flow filed and reveal the major physical features of the flow.

As the experiment data measured at stator leading edge are 
not available, comparisons of radial profiles could only be performed between the throughflow calculation and the $3 \mathrm{D}$ CFD computation. Figure 6 shows the radial profiles of the total pressure recovery coefficient of the IGV. The profile predicted by Euler and N-S throughflow solutions agree well with that obtained from 3D CFD result along the entire blade span except the near-endwall regions, where both throughflow calculations predict a higher loss than 3D CFD calculation does. Furthermore, the N-S throughflow solution agrees much better with the $3 \mathrm{D}$ viscous result than Euler throughflow solution in tip region, which demonstrates the advantage of the viscous throughflow model.

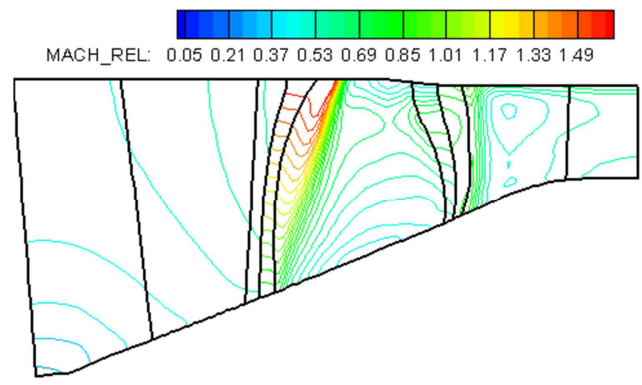

Figure 3. Contours of the relative Mach number calculated by the Euler throughflow model.

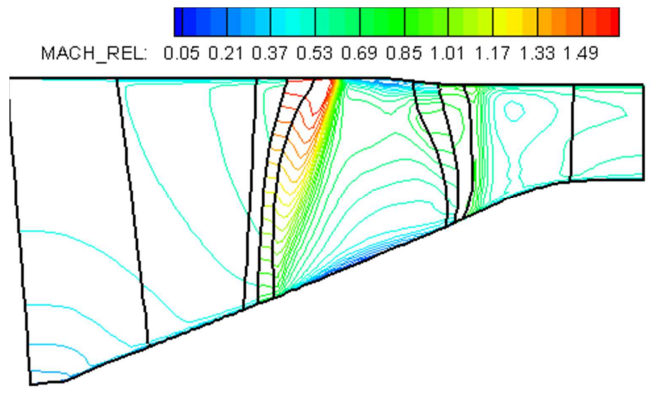

Figure 4. Contours of the relative Mach number calculated by the N-S throughflow model.

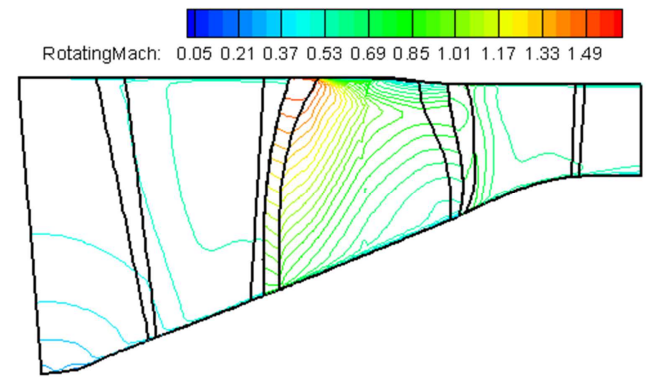

Figure 5. Contours of the $3 D$ circumferentially averaged relative Mach number.

Figure 7 to Figure 9 show the radial profiles of the total pressure ratio, total temperature ratio and the outlet relative flow angle of the rotor. Both two throughflow methods predict a higher pressure ratio in the region above $60 \%$ span and a lower pressure ratio in the region under $40 \%$ span than 3D CFD method does. The pressure ratio calculated by N-S throughflow model is higher in near tip region and lower in near hub region compared to the result from Euler throughflow model.

Figure 8 shows that N-S throughflow overestimates temperature ratio in the near tip region, and Figure 9 indicates that the deviation angle is overestimated in the region under $20 \%$ span and underestimated in the region above $40 \%$ span by both Euler and N-S throughflow methods.

There are close connections between the deviation angle and the work done by the rotor, and the underestimation of deviation angle is therefore one of the major reasons why both throughflow models predict a higher total temperature ratio and total pressure ratio. Another reason is that throughflow models do not incorporate leakage effects of tip clearance.

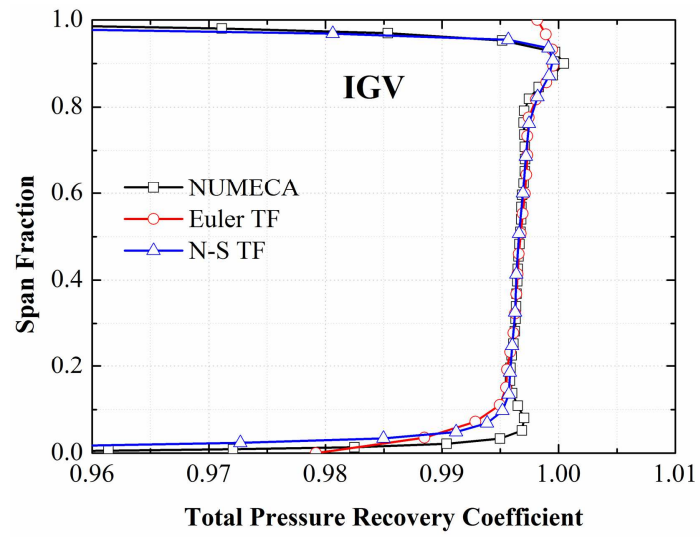

Figure 6. Total pressure recovery coefficient of the IGV.

Figure 10 and Figure 11 compare the radial profiles of the total pressure recovery coefficient and the outlet flow angle of the stator respectively. Results of Euler and N-S throughflow methods are almost the same along the whole span except hub and tip region. The N-S throughflow model predicts much lower pressure recovery coefficient in hub and tip region, which clearly shows the effect of annulus boundary layer. Figure 10 shows that result of N-S throughflow method is almost the same as the result of $3 \mathrm{D}$ CFD calculation in hub region, and then begins to diverge where the span is above $20 \%$. There is little difference between the outlet absolute flow angle predicted by Euler throughflow and N-S throughflow method, both are close to 3D CFD results.

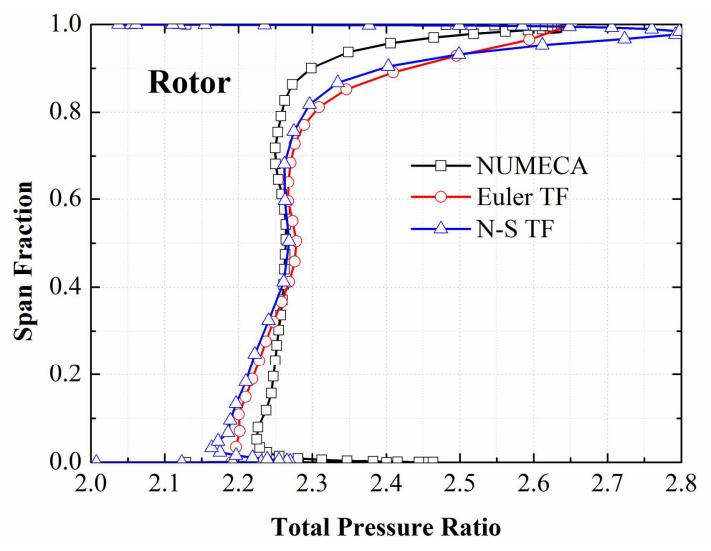

Figure 7. Total pressure ratio of the rotor. 


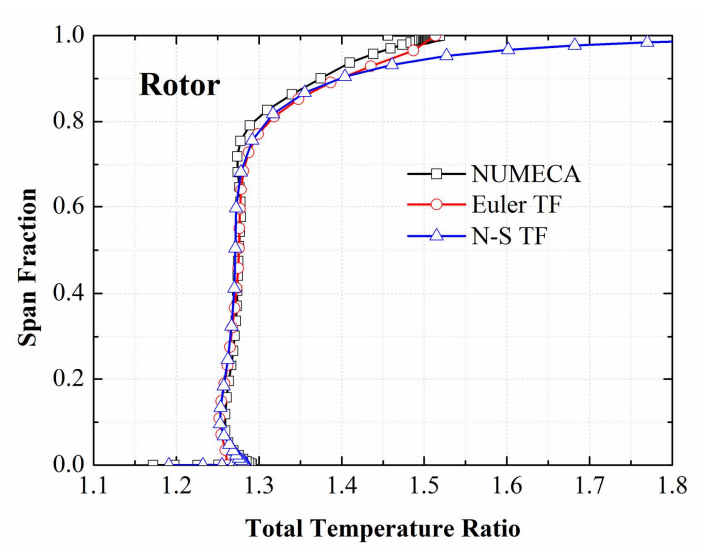

Figure 8. Total temperature ratio of the rotor.

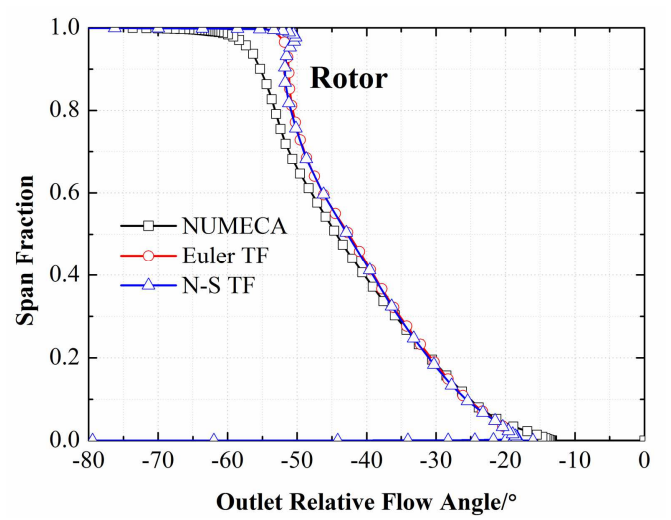

Figure 9. Relative flow angle at the outlet of the rotor.

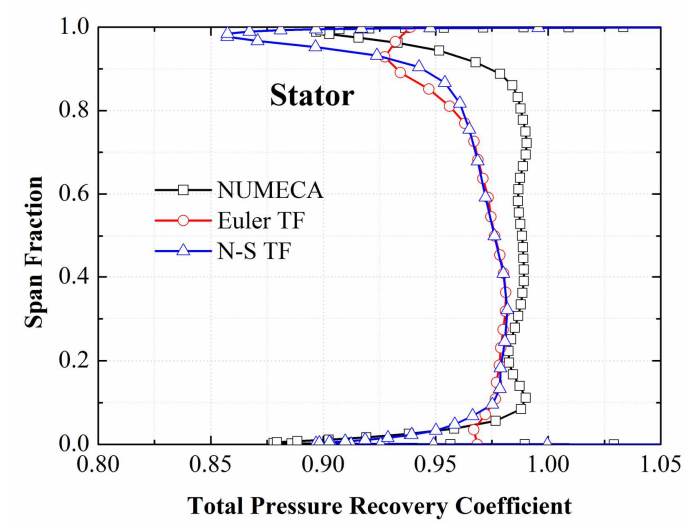

Figure 10. Total pressure recovery coefficient of the stator.

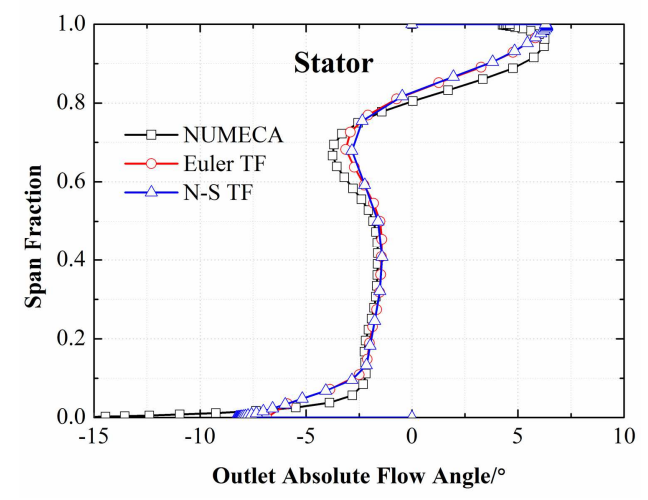

Figure 11. Absolute flow angle at the outlet of the stator.

\section{Conclusions}

A new viscous throughflow model has been developed based on a previous inviscid throughflow model. The governing equations are derived by circumferentially averaging Navier-Stokes equations combined with the Spalart-Allmaras turbulence model. These equations are solved by a time-marching finite volume method.

To validate this model, this new throughflow model is then applied to predict the performance of a 1.5 stage fan, and the predicted performance is compared to the experiment data. The meridional flow field and radial profiles generated by this model are also compared with those obtained through Numeca software.

The agreements between throughflow analysis and experiment data are reasonably good. The throughflow model can predict quite similar flow patterns and radial distributions of some parameters compared to Numeca software, which means that this throughflow model can reveal the major physical features of the flow. However, there are noticeable differences in values, despite the similar radial distribution. The further step is to improve the accuracy of loss and deviation angle prediction.

\section{Nomenclatures}

\begin{tabular}{|c|c|}
\hline$x, r, \varphi$ & Axial, radial and circumferential coordinates \\
\hline$\rho$ & Density \\
\hline$p$ & Pressure \\
\hline$T$ & Temperature \\
\hline$w$ & Relative velocity \\
\hline E & Total internal energy \\
\hline$H$ & Total enthalpy \\
\hline$\tilde{v}$ & Solution variable in S-A turbulence model \\
\hline$b$ & Blockage factor \\
\hline$N$ & Number of blades \\
\hline$\omega$ & Rotating speed \\
\hline$U$ & Vector of conservative variables \\
\hline$F, F_{v}, G, G_{v}$ & Vectors of convective fluxes \\
\hline$S$ & Vector of source terms \\
\hline$F_{\mathrm{b}}$ & Inviscid blade force \\
\hline$F_{F}$ & Viscous blade force \\
\hline$n$ & Unit normal vector \\
\hline$\tau_{i j}$ & Viscous stress \\
\hline$q_{x}, q_{r}$ & Heat flux \\
\hline \multicolumn{2}{|l|}{ Subscripts } \\
\hline$x, r, \varphi$ & Axial, radial and circumferential coordinates \\
\hline$p$ & Pressure surface \\
\hline$S$ & Suction surface \\
\hline$B$ & Inviscid blade force \\
\hline$F$ & Viscous blade force \\
\hline tb & Turbulence model \\
\hline
\end{tabular}




\section{Acknowledgements}

This work is sponsored by Aeronautical Science Foundation of China (No. 2015ZB08006).

\section{References}

[1] L. H. Smith Jr., "The radial-equilibrium equation of turbomachinery," Journal of Engineering for Power, Transactions of the ASME, Vol. 88, No. 1, 1966.

[2] R. A. Novak, "Streamline curvature computing procedures for fluid-flow problems," Journal of Engineering for Power, Transactions of the ASME, Vol. 89, No. 4, 1967.

[3] A. Spurr, "The prediction of 3D transonic flow in turbomachinery using a combined throughflow and blade-toblade time marching method," International Journal of Heat Fluid Flow, Vol. 2, No. 4, 1980, pp. 189-199.

[4] Dingxi Wang, "Turbomachinery aerodynamic and aeromechanic design optimization using the adjoint method," Ph. D. thesis, University of Durham, 2008.

[5] S. Baralon, L-E. Erikson, and U. Hall, "Validation of a throughflow time-marching finite-volume solver for transonic compressors," ASME Paper 98-GT-47.

[6] S. Baralon, L-E. Erikson, and U. Hall, "Evaluation of highorder terms in the throughflow approximation using 3D Navier-Stokes computations of a transonic compressor rotor," ASME Paper 99-GT-74.

[7] S. Baralon, L-E. Erikson, and U. Hall, "Viscous throughflow modelling of transonic compressors using a time-marching finite-volume solver," 13th International Symposium on Airbreathing Engines (ISABE), Chattanooga, USA, 1997.

[8] J. Simon and O. Leonard, "A throughflow analysis tool based on the Navier-Stokes equations," Proceedings of the 6th European Turbomachinery Conference, Lille, 2005.

[9] A. Sturmayr, C. Hirsch, "Shock representation by Euler throughflow models and comparison with pitch-averaged Navier-Stokes solutions," ISABE 99-7281.

[10] R. A. Gentry, R. E. Martin, and B. J. Daly, "An Eulerian differencing method for unsteady compressible flow problems," Journal of Computational Physics, Vol. 1, 1966.

[11] F. F. Ning, "Numerical investigations of flows in transonic compressors with real geometrical complexities," $\mathrm{Ph}$. D. thesis, Beijing University of Aeronautics and Astronautics, 2002.

[12] C. Bosman, and H. Marsh, "An improved method for calculating the flow in turbo-machines, including a consistent loss model," Journal of Mechanical Engineering Sciences, Vol. 16, 1974, pp. 25-31.

[13] Miller, D. C., Wasdell, D. L.. "Off-design prediction of compressor blade losses,” I. Mech. E., C279/87, 1987.

[14] Wright, P. I., Miller, D. C., "An improved compressor performance prediction model” I. Mech. E. C423/028, 1991.

[15] Dunham, J., "Compressor off-design performance prediction using an endwall model, " ASME Paper, GT1996-62, 1996.

[16] Hailiang Jin, Donghai Jin, Xingmin Gui, “A time-marching throughflow model and its application in transonic axial compressor", Journal of Thermal Science volume 19, pages519-525 (2010).

[17] J. R. Edwards, "A low-diffusion flux-splitting scheme for Navier-Stokes calculations," Computers \& Fluids, Vol. 26, No 6, 1997, pp. 635-659.

[18] Jameson, A., Schimidt, W.. Turkel, E., "Numerical solutions of the euler equations by finite volume methods with RuneKutta time stepping schemes,” AIAA Paper, 81-1259, 1981. 